\title{
Unruptured Paraclinoid Carotid Aneurysms Occur More Frequently in Younger Ages
}

\author{
Reo Kawaguchi, MD, PhD, Shigeru Miyachi, MD, PhD, Tomotaka Ohshima, MD, PhD, \\ Naoki Matsuo, MD, PhD \\ Department of Neurological Surgery and Neuroendovascular Therapy Center, Aichi Medical University, Aichi, Japan
}

Purpose: We investigated the age distribution of cerebral saccular aneurysms in various locations to clarify the differences by location and discuss the mechanism of formation.

Materials and Methods: We retrospectively assessed clinical material obtained from 1,252 unruptured aneurysms treated with endovascular embolization between 2004 and 2019. Age, sex, laterality, and size were investigated by the location of aneurysms, classified as cavernous internal carotid artery (ICA), paraclinoid ICA, supraclinoid ICA, anterior communicating artery, anterior cerebral artery, middle cerebral artery, basilar artery complex, and posterior inferior cerebellar artery. Paraclinoid aneurysms were subclassified into 3 patterns according to their projecting direction: S-type, with superior protrusion; M-type, with medial protrusion; and P-type, with posteroinferior protrusion.

Results: There was no significant difference by location for sex, laterality, and size. The mean age of patients with paraclinoid aneurysms (56.5 years old) was significantly lower than that of other aneurysm patients (64.3 years old). Notably, $40 \%$ of the patients with M-type aneurysms were $<50$ years old. This percentage was significantly higher than that of aneurysms at other locations $(P<0.05)$.

Conclusion: We found a young female predominance for patients with paraclinoid carotid aneurysms. This study may suggest that congenital factors contribute to paraclinoid aneurysm formation as well acquired factors, such as hemodynamic stress, atherosclerotic wall damage, and local inflammation.

Key Words: Cerebral aneurysm; Internal carotid artery; Paraclinoid; Age; Sex

\section{INTRODUCTION}

Development in imaging technologies has resulted in increased requests for magnetic resonance angiography imaging of the brain, which has contributed to an increased rate of detection of unruptured aneurysms. According to Japanese studies on treatment indications for unruptured aneurysms, curative treatment is allowed for patients with a life expectancy $>10$ years with an aneurysm with a maximum diameter of 5-7 $\mathrm{mm} \mathrm{r}^{1,2}$ Aneurysms with a mass effect, a high-risk configuration like an irregular shape or bleb formation, or a posterior circulation location like the posterior communicating artery (PComA) can also be considered for treatment. ${ }^{3}$ According to Fischer's classification, ${ }^{4,5}$ paraclinoid

\section{Correspondence to:}

Shigeru Miyachi, MD, PhD

Department of Neurological Surgery and Neuroendovascular Therapy Center, Aichi Medical University, 1-1, Yazakokarimata, Nagakute 480-1195, Aichi, Japan

Tel: +81-561-62-3311

Fax: +81-561-62-2879

E-mail: miyachi.shigeru.752@mail. aichi-med-u.ac.jp

Received: January 25, 2021

Revised: April 17, 2021

Accepted: April 26, 2021
Copyright $\odot 2021$ Korean Society of Interventional Neuroradiology This is an Open Access article distributed under the terms of the Creative Commons Attribution Non-Commercial License (http://creativecommons.org/licenses/by-nc/4.0) which permits unrestricted non-commercial use, distribution, and reproduction in any medium, provided the original work is properly cited.

pISSN 2093-9043 eISSN 2233-6273 
aneurysms occur at the $\mathrm{C} 2-\mathrm{C} 3$ portion of the internal carotid artery (ICA) and include aneurysms at the carotid cave, superior hypophyseal artery (SHA), ophthalmic artery, and anterior wall (dorsal) of the intracranial ICA. Since these aneurysms are at low risk of rupture, ${ }^{1,6}$ they have not been the focus of surgical treatments, except for ruptured blood blister-like aneurysms or large symptomatic ophthalmic aneurysms. However, because more young people are becoming health-oriented, the performance of magnetic resonance imaging (MRI) check-ups has increased, resulting in an increased detection of middle-sized paraclinoid aneurysms. When looking over the data of these patients, we noticed an unusually high frequency of the detection of paraclinoid aneurysms in young people. Therefore, we reviewed our 16-year patient series of endovascularly-treated unruptured aneurysms to prove this tendency statistically by comparing our observation with aneurysms at other locations and discuss the reason for this from an embryological point of view.

\section{MATERIALS AND METHODS}

The authors retrospectively reviewed 1,252 cases of unruptured aneurysms treated with endovascular treatment in the hospitals of Nagoya University, Osaka Medical College, and Aichi Medical University, Japan, between January 2004 and December 2019. This study was a retrospective review of anonymous data to collect only the age of patients and imaging data from our database. We excluded aneurysms treated with surgical clipping or those conservatively observed, since there was no detailed data to define the exact classification. We focused on the patient profile (age and sex) and aneurysm profile (location, laterality, and size) obtained from the clinical records. The data, particularly age and aneurysm size, was collected at the time of proper diagnosis by angiographic imaging. We did not consider details such as why the aneurysm was found (for example, during a brain checkup), the coincidental detection of other ruptured aneurysms, minute examination for head trauma, or the diagnosis as the cause of headaches, tinnitus, and other neurological disorders. Although some of these aneurysms were very small, less than $5 \mathrm{~mm}$ in size, they were mostly coincidental aneurysms with those in other lesions and were treated together. This study was approved by the respective Institution Review Boards, and consent was obtained from all patients using simple consent forms. Privacy protection was achieved for each patient by not harvesting private information from the patient profile that would specify their individuality.

The location of the saccular aneurysms was grossly classified according to the usual manner ${ }^{1}$ as cavernous ICA, paraclinoid ICA, supraclinoid ICA, anterior communicating artery (AComA), anterior cerebral artery (ACA), middle cerebral artery (MCA), basilar artery (BA) complex, or posterior inferior cerebellar artery (PICA). Paraclinoid ICA aneurysms were specifically subclassified into 3 categories based on the surgical classification ${ }^{7,8}$ as below (Fig. 1A). Fusiform aneurysms and dissecting aneurysms were excluded from this study.

Anterior circulation aneurysms included cavernous ICA, paraclinoid ICA (including carotid cave aneurysm, aneurysms at the SHA and ophthalmic artery, and anterior wall aneurysms), supraclinoid ICA (including aneurysms at the PComA, anterior choroidal artery, and ICA terminal aneurysms), ACo$m A$ (including A1 aneurysms), distal ACA, and MCA bifurcation aneurysms.

Posterior circulation aneurysms included BA complex (including terminal, superior cerebellar artery, and trunk aneurysms) and vertebral artery-PICA. Paraclinoid ICA aneurysms were classified into 3 types. The superior projecting type (S-type) comprised aneurysms that projected superiorly with the neck at the origin of the ophthalmic artery and more distal C2 portion, including the ophthalmic artery aneurysm and ICA anterior wall (dorsal) aneurysm. The medial pro-

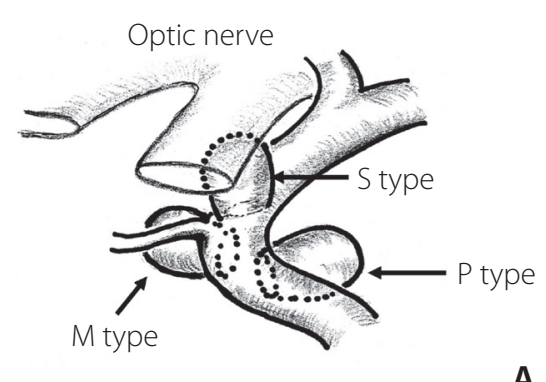

A

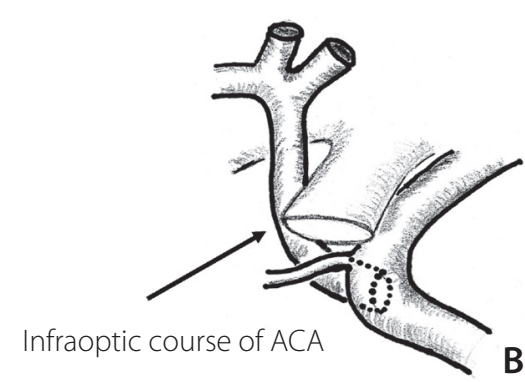

Fig. 1. Schemes of 3 types of paraclinoid carotid aneurysms. S-type, superior projecting type; M-type, medial projecting type; P-type, posterior projecting type (A). Note that the location of M-type aneurysms seems to correspond well with the site of anastomosis of primitive ophthalmic arteries or the persistent infraoptic course of the anterior cerebral artery (ACA) (B). 
jecting type (M-type) comprised aneurysms that protruded medially from the intradural neck of the anterior genu to the carotid siphon (proximal C2), including ICA-SHA aneurysms. The posterior projecting type (P-type) comprised aneurysms with posteroinferior protrusion from the infraclinoid neck at the inner curve of the $\mathrm{C} 3$ genu portion, including infraclinoid extradural carotid cave aneurysms. Intermediate-type aneurysms were categorized based on a more superior projecting direction or neck position. In the classification of ophthalmic segment aneurysms, ${ }^{9}$ M-type and P-type correspond to suprasellar and paraclinoid variants, respectively. Assignment of cases based on this subclassification was performed by multiple neurointerventionists.

We did not investigate patient-specific factors that could have influenced the data deviation, such as family history and the presence of congenital connective tissue disease and vascular malformations. We also did not investigate aneurysm-specific factors, such as multiplicity and history of growth. This study was based on retrospective observations of angiographic images of the patients.

Statistical analysis was performed using SPSS 25.0 statistical software package (IBM Co., Armonk, NY, USA). Data are presented as means for continuous variables and as frequencies (percentages) for categorical variables. A P-value $\leq 0.05$ was considered statistically significant.

\section{RESULTS}

We retrospectively assessed 1,252 aneurysms in 1,221 patients who underwent endovascular treatment. Patients with multiple aneurysms in different locations were counted by number in each category, and ones with aneurysms included in the same location like kissing aneurysms were counted as 1 patient. The average age of the patients was 61.8 years old (range, 27-91 years), and 75\% of the patients were female. The angiographic, anatomic, and demographic characteristics, as well as the aneurysmal profile, are described in Table 1. Sex distribution was predominantly female (>65\%) in all locations, except for AComA and MCA aneurysms. Although the laterality showed a slight left dominant tendency and small differences by location, this was not statistically significant. The mean aneurysm size was $7.6 \mathrm{~mm}$ (range, 2-40 mm) and was particularly larger in carotid cavernous aneurysms (mean, $13.5 \mathrm{~mm}$ ) because this group included a large number of giant aneurysms treated with a flow diverter. The age distribution of patients with paraclinoid aneurysms was significantly lower (mean, 56.5 years old) than that of other aneurysm

Table 1. Angiographic, anatomic, and demographic characteristics and aneurysmal profile of 1,252 aneurysms in 1,221 patients who underwent endovascular treatment

\begin{tabular}{|c|c|c|c|c|c|c|}
\hline Type of aneurysm & $\begin{array}{c}\text { Number of } \\
\text { patients }\end{array}$ & Age (yr) & $\begin{array}{c}\text { Age under } 50 \\
\text { years old }\end{array}$ & Sex, female (\%) & $\begin{array}{l}\text { Laterality, } \\
\text { left side (\%) }\end{array}$ & $\begin{array}{c}\text { Aneurysm size } \\
(\mathrm{mm})\end{array}$ \\
\hline ICA (paraclinoid) ${ }^{*}$ & 402 & $56.5(27-81)$ & $123(30.6)$ & 80.3 & 58.0 & $7.3(2-30)$ \\
\hline M-type & 198 & $54.4(27-81)$ & $79(40.0)$ & 81.3 & 61.1 & $6.2(2-20)$ \\
\hline S-type & 151 & $57.0(27-80)$ & $42(27.8)$ & 80.1 & 59.6 & $8.1(2-25)$ \\
\hline P-type & 53 & $63.2(50-79)$ & $2(3.8)$ & 77.4 & 43.4 & $9.0(4-30)$ \\
\hline ICA (cavernous) & 148 & $63.4(27-90)$ & $27(18.2)$ & 86.5 & 53.7 & $13.5(4-40)$ \\
\hline ICA (supraclinoid) & 233 & $63.0(32-91)$ & $41(17.6)$ & 79.0 & 54.1 & $6.7(2-24)$ \\
\hline AComA complex & 155 & $64.1(34-88)$ & $13(8.4)$ & 48.4 & ND & $5.8(3-14)$ \\
\hline Distal ACA & 35 & $67.1(39-81)$ & $2(5.7)$ & 71.4 & 60.0 & $5.7(2-10)$ \\
\hline MCA & 75 & $60.0(37-79)$ & $12(16.0)$ & 57.3 & 41.9 & $5.5(3-11)$ \\
\hline BA complex & 166 & $63.8(31-88)$ & $17(10.2)$ & 70.5 & $53.5^{\dagger}$ & $7.9(2-25)$ \\
\hline PICA & 38 & $60.7(40-81)$ & $6(15.8)$ & 65.8 & 64.9 & $5.4(3-12)$ \\
\hline Total & 1,252 & $61.8(27-91)$ & $241(19.5)$ & 75.0 & 55.10 & $7.6(2-40)$ \\
\hline
\end{tabular}

Values are presented as mean (range) or number (\%).

ICA, internal carotid artery; AComA, anterior communicating artery; ACA, anterior cerebral artery; MCA, middle cerebral artery; BA, basilar artery; PICA, posterior inferior cerebellar artery; S-type, superior projecting type; M-type, medial projecting type; P-type, posterior projecting type; ND, not determined.

* See the text about subclassification. ${ }^{\dagger}$ Excluding data for BA terminal aneurysms. 
patients (mean, 64.3 years old). Furthermore, $>30 \%$ of paraclinoid aneurysm patients were under 50 years old. Notably, $40 \%$ of the patients with M-type aneurysms were under 50 years old. This percentage was significantly higher than that of aneurysms in other locations $(P<0.05)$. The S-type aneurysm group also showed a tendency toward a younger age distribution. In contrast, the age of the patients with P-type aneurysms was widely distributed.

\section{DISCUSSION}

Unruptured carotid aneurysms are coincidentally found in brain check-ups or in MRI examinations for other neurological disorders, with paraclinoid aneurysms being the most frequently observed (approximately one-third of coincidental aneurysms). However, because of their low rupture risk, incidental paraclinoid aneurysms $<10 \mathrm{~mm}$ in diameter have often been observed without treatments, such as asymptomatic carotid cavernous aneurysms. ${ }^{6}$ Previous reports showed a female predominance in patients with paraclinoid aneurysms, ${ }^{10}$ but there have been no papers that refer to age distribution. In this study, paraclinoid aneurysms tended to be found in younger patients. If we consider that the mechanism of growth and speed is equal in all aneurysms, younger patients should have a higher risk of rupture because they have a long period of their lives to live. However, the rate of rupture of M-type aneurysms is actually low. This discrepancy may not be explained by acquired factors causing aneurysms like hemodynamic stress, atherosclerotic wall damage, and local inflammation."

When considering the anatomical situation of aneurysms, they usually arise from the bifurcation of the main trunk of cerebral arteries or at the origin of the main branches. However, most paraclinoid aneurysms are independent from the big branches except for the ophthalmic aneurysms. Although SHA is one of the branches of the carotid artery, it is too tiny to visualize even on a selective angiogram. Further, Tanaka et al. ${ }^{12}$ described that $20 \%$ of paraclinoid aneurysms are associated with no branching arteries. Thus, paraclinoid aneurysms have specific structural characteristics.

On the embryological point of view, the paraclinoid portion of the ICA is the specific site of drastic change during branching of the ophthalmic artery in the embryological period. According to a theory on the development of ophthalmic artery, the primitive ophthalmic artery is initially formed from 2 arteries, namely, the ventral ophthalmic artery originating from the ACA and the dorsal ophthalmic artery originating from the $\mathrm{C} 4$ portion of the ICA, when the embryo is $4-8 \mathrm{~mm}$ long. These 2 arteries run medial to the siphon and establish anastomosis on the inner aspect of the siphon at the sellar level. ${ }^{13,14}$ When the embryo reaches 40 $\mathrm{mm}$ in length, the stapedial artery anastomoses to the primitive ophthalmic artery. Finally, the supraorbital portion of the ventral ophthalmic artery regresses, resulting in a complete normal adult-type ophthalmic artery. However, if this anastomosis of the ventral ophthalmic artery remains, the infraoptic course of the ACA persists (Fig. 1B). ${ }^{13,15}$ Thus, during the development process of the ophthalmic artery and ACA, this dynamic migration and regression will cause structural fragility. In fact, various congenital anomalies and persistent variants have been reported around this area. ${ }^{13}$

There is other evidence that the persistence of the primitive arteries often causes a higher rate of aneurysm formation. Lasjaunias et al mentioned the proximal duplication of the ACA is as frequent as $8 \%$ in patients with aneurysms. ${ }^{13}$ Furthermore, abnormally frequent aneurysm formation at the origin of a persistent primitive artery, particularly aneurysms at persistent trigeminal arteries ${ }^{16}$ and persistent hypoglossal arteries, ${ }^{17}$ are well known. Interestingly, these reports suggest that such specific aneurysms on an arterial anomaly are found in patients of a relatively younger age. 18,19 Some reports have demonstrated cases in patients under 30 years old. ${ }^{19,20}$ Chalouhi et al. ${ }^{10}$ pointed out a high frequency of multiple aneurysms in patients with paraclinoid aneurysms, and it may support the congenital fragility of the arterial wall. Tanaka ${ }^{21}$ reported a case of a paraclinoid aneurysm at a primitive dorsal ophthalmic artery. This interesting case may show aneurysmal formation at the trace remains of the anastomotic portion of the primitive ventral ophthalmic artery to the paraclinoid portion. Considering our result, in which paraclinoid aneurysms, particularly the M-type, were found in an unusually younger generation, congenital factors may be responsible for their formation mechanism.

Since is a retrospective study of only treated cases, the subjects do not reflect all patients with conservatively observed or occult aneurysms. However, because the sample size is relatively large and this was a multi-center study over a long period, factors due to arbitrary deviation or oversight might have been neglected. Furthermore, the age at which the concerned aneurysm is found does not equate with the moment of birth of the aneurysm. It has not been determined 
how the exact length of existence of unruptured aneurysms can be calculated.

Although familial history was not thoroughly investigated in our study, gene-related factors might have been involved in the aneurysm formation process. There are some reports showing that aneurysms in patients with a corresponding family history tend to be found in the earlier years, instead of having a sporadic age distribution. ${ }^{22}$ Additionally, an experimental study using a rat model of bifurcation aneurysms suggested the involvement of gene deficiency as well as hemodynamic stress and local inflammation. ${ }^{23}$ Further multi-axial and larger epidemiologic studies with big data and histopathological confirmation are crucial. However, the characteristic age deviation for this specific aneurysm might suggest consideration of the mechanism of aneurysm formation.

\section{CONCLUSION}

We retrospectively investigated the age distribution of cerebral aneurysms and found that unruptured, medially protruding paraclinoid carotid aneurysms were significantly predominant in younger patients. This tendency may be due to the influence of the embryological factors resulting in the local fragility of the carotid wall during the embryological period. The characteristic age deviation for this specific aneurysm might suggest consideration of the mechanism of aneurysm formation.

\section{Fund}

None.

\section{Ethics Statement}

This study was approved by the respective Institution Review Boards, and consent was obtained from all patients using simple consent forms.

\section{Conflicts of Interest}

The authors have no conflicts to disclose.

\section{Author Contributions}

Concept and design: SM. Analysis and interpretation: RK and SM. Data collection: RK, SM, TO, and NM. Writing the article: RK. Critical revision of the article: SM. Final approval of the article: SM. Statistical analysis: RK. Overall responsibility: SM.

\section{ORCID}

Reo Kawaguchi: https://orcid.org/0000-0003-2619-0765

Shigeru Miyachi: https://orcid.org/0000-0003-2703-1379

Tomotaka Ohshima: https://orcid.org/0000-0002-4810-1703

Naoki Matsuo: https://orcid.org/0000-0001-8346-1627

\section{REFERENCES}

1. UCAS Japan Investigators, Morita A, Kirino T, Hashi K, Aoki N, Fukuhara $S$, et al. The natural course of unruptured cerebral aneurysms in a Japanese cohort. NEng/ J Med 2012;366:2474-2482

2. Sonobe M, Yamazaki T, Yonekura M, Kikuchi H. Small unruptured intracranial aneurysm verification study: SUAVe study, Japan. Stroke 2010;41:1969-1977

3. Brain Dock guidelines 2008 [Internet]. Sapporo: The Japan Brain Dock Society [cited 2019 Mar 20]. Available from: http://jbds.jp/ doc/guideline2008.pdf

4. Fischer E. Die Lageabweichungen der vorderen hirnarterie im gefassbild. Zentralb/ Neurochir 1938;3:300-313

5. Bouthillier A, van Loveren HR, Keller JT. Segments of the internal carotid artery: a new classification. Neurosurgery 1996;38:425432

6. Greving JP, Wermer MJ, Brown RD Jr, Morita A, Juvela S, Yonekura $\mathrm{M}$, et al. Development of the PHASES score for prediction of risk of rupture of intracranial aneurysms: a pooled analysis of six prospective cohort studies. Lancet Neurol 2014;13:59-66

7. Kyoshima K, Koike G, Hokama M, Toriyama T, Gibo H, Okudera $\mathrm{H}$, et al. A classification of juxta-dural ring aneurysms with reference to surgical anatomy. J Clin Neurosci 1996;3:61-64

8. Kobayashi S, Kyoshima K, Gibo H, Hegde SA, Takemae T, Sugita K. Carotid cave aneurysms of the internal carotid artery. J Neurosurg 1989;70:216-221

9. Day AL. Aneurysms of the ophthalmic segment. A clinical and anatomical analysis. J Neurosurg 1990;72:677-691

10. Chalouhi N, Tjoumakaris S, Dumont AS, Gonzalez LF, Randazzo C, Gordon D, et al. Superior hypophyseal artery aneurysms have the lowest recurrence rate with endovascular therapy. AJNR Am J Neuroradiol 2012;33:1502-1506

11. Huttunen $T$, von und zu Fraunberg M, Frösen J, Lehecka M, Tromp G, Helin K, et al. Saccular intracranial aneurysm disease: distribution of site, size, and age suggests different etiologies for aneurysm formation and rupture in 316 familial and 1454 sporadic eastern Finnish patients. Neurosurgery 2010;66:631-638

12. Tanaka Y, Hongo K, Tada T, Nagashima H, Horiuchi T, Goto T, et al. Radiometric analysis of paraclinoid carotid artery aneurysms. 
J Neurosurg 2002;96:649-653

13. Lasjaunias P, Berenstein A, ter Brugge KG. Intradural arteries. In: Lasjaunias P, Berenstein A, ter Brugge KG. Surgical neuroangiography: clinical vascular anatomy and variations, 2 nd ed. Heidelberg: Springer, 2001;479-629

14. Komiyama M. Letter to the editor - embryology of the ophthalmic artery: a revived concept. Interv Neuroradiol 2009;15:363368

15. Nutik S, Dilenge D. Carotid-anterior cerebral artery anastomosis. Case report. J Neurosurg 1976;44:378-382

16. Murai S, Sugiu K, Hishikawa T, Hiramatsu M, Nishihiro S, Kidani $\mathrm{N}$, et al. Endovascular treatment for unruptured aneurysm associated with persistent primitive trigeminal artery: a case report and literature review. Acta Neurochir (Wien) 2019;161:407-411

17. Varvari I, Bos EM, Dinkelaar W, van Es AC, Can A, Hunfeld M, et al. Fatal subarachnoid hemorrhage from an aneurysm of a persistent primitive hypoglossal artery: case series and literature overview. World Neurosurg 2018;117:285-291

18. Zenteno M, Lee A, Moscote-Salazar LR. Rupture of persistent primitive trigeminal artery-basilar artery aneurysm managed with stent-assisted coiling. Asian J Neurosurg 2018;13:817-821

19. Ishikawa T, Yamaguchi K, Anami H, Sumi M, Ishikawa T, Kawamata T. Treatment of large or giant cavernous aneurysm associated with persistent trigeminal artery: case report and review of literature. World Neurosurg 2017;108:996.e11-996.e15

20. Kimball D, Ples H, Miclaus GD, Matusz P, Loukas M. Persistent hypoglossal artery aneurysm located in the hypoglossal canal with associated subarachnoid hemorrhage. Surg Radiol Anat 2015;37:205-209

21. Tanaka M. Persistent primitive dorsal ophthalmic artery associated with paraclinoid internal carotid artery aneurysm. J Neuroendovasc Ther 2009;3:39-41

22. Aoki T, Nishimura M. The development and the use of experimental animal models to study the underlying mechanisms of CA formation. J Biomed Biotechnol 2011;2011:535921

23. Aoki T, Miyata H, Abekura Y, Koseki H, Shimizu K. Rat model of intracranial aneurysm: variations, usefulness, and limitations of the Hashimoto model. Acta Neurochir Supp/ 2020;127:35-41 